\title{
Ring-opening metathesis and ring-closing metathesis of bicyclo[4.2.0]octene-ynes: application to the synthesis of tricyclic compounds
}

\author{
Hideaki Wakamatsu,* Satoko Hareyama, and Mitsuhiro Takeshita \\ Tohoku Pharmaceutical University, 4-4-1 Komatsushima, Aoba-ku, Sendai 981-8558, Japan \\ E-mail: hiwaka@tohoku-pharm.ac.jp
}

Dedicated to Prof. S. Blechert on the occasion of his $65^{\text {th }}$ birthday

\begin{abstract}
Ring-opening metathesis and ring-closing metathesis (ROM-RCM) of bicyclo[4.2.0]octene-ynes and their application to the synthesis of tricyclic derivatives have been demonstrated using a second-generation ruthenium carbene complex. When bicycloalkene having a propargylamino group as an alkyne tether was reacted with a second-generation ruthenium carbene complex under an ethylene atmosphere, ROM-RCM proceeded to give tricyclic heterocycles in good yield. On the other hand, when the effect of the substituent on the alkyne was examined, cross metathesis (CM) of the alkyne part with ethylene proceeded to provide a conjugated diene derivative.
\end{abstract}

Keywords: Ring-opening metathesis and ring-closing metathesis, ruthenium, carbene complex, cross metathesis, ethylene, cycloalkene-yne

\section{Introduction}

Olefin metathesis contains a cleavage of carbon-carbon double bonds concomitantly with the formation of other ones by a metal carbene complex. ${ }^{1}$ Currently, it has become a powerful synthetic method for the formation of carbon-carbon double bonds in the field of synthetic organic chemistry. Enyne metathesis, ${ }^{2}$ which takes place between a double bond and triple bond, is of particular interest. The diene derivative is obtained by enyne metathesis, although a twocarbon unit is thrown away as an ethylene by olefin metathesis. When enyne metathesis is carried out as an intramolecular reaction, the olefin part of enyne is cleaved and its alkylidene part is transferred to an alkyne. As a result, a cyclized diene derivative is obtained (Scheme 1). 


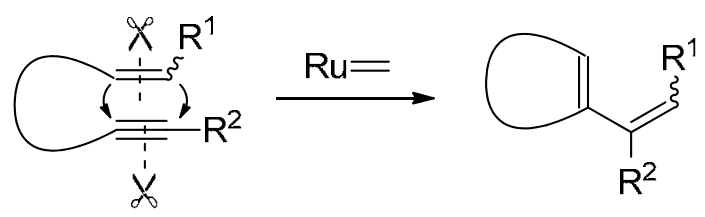

Scheme 1. Enyne metathesis.

After the first report of enyne metathesis by Katz and co-workers, ${ }^{3}$ the progressive results, which contain the total synthesis of stemoamide ${ }^{2 \mathrm{~g}}$ and the effect of ethylene, ${ }^{2 \mathrm{f}}$ were reported. ${ }^{2}$ Recently, we developed ROM-RCM of cycloalkene-ynes. ${ }^{4,5}$ When a metathesis reaction of cycloalkene-yne, whose tether having an alkyne part is connected to the C-1 position of cycloalkene, was carried out in the presence of $\mathbf{1}$ under an ethylene atmosphere, ROM-RCM proceeded smoothly to provide bicyclic compound 3 in good yield (Scheme 2).

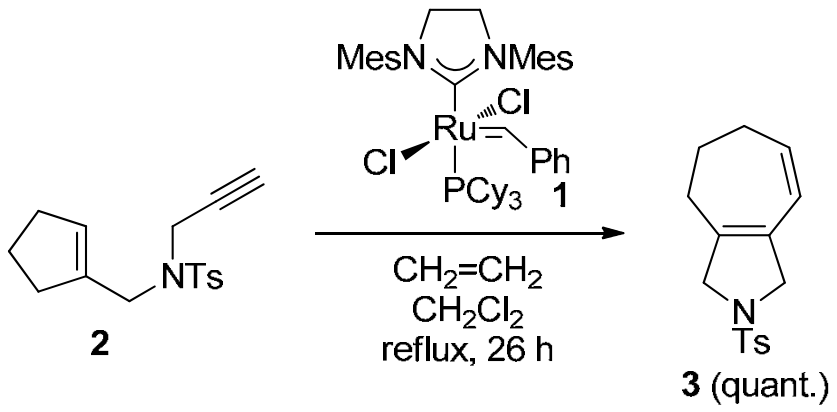

Scheme 2. Ring-Opening Metathesis and Ring-Closing Metathesis of Cycloalkene-ynes.

The reaction mechanism of ROM-RCM is shown in Scheme 3.
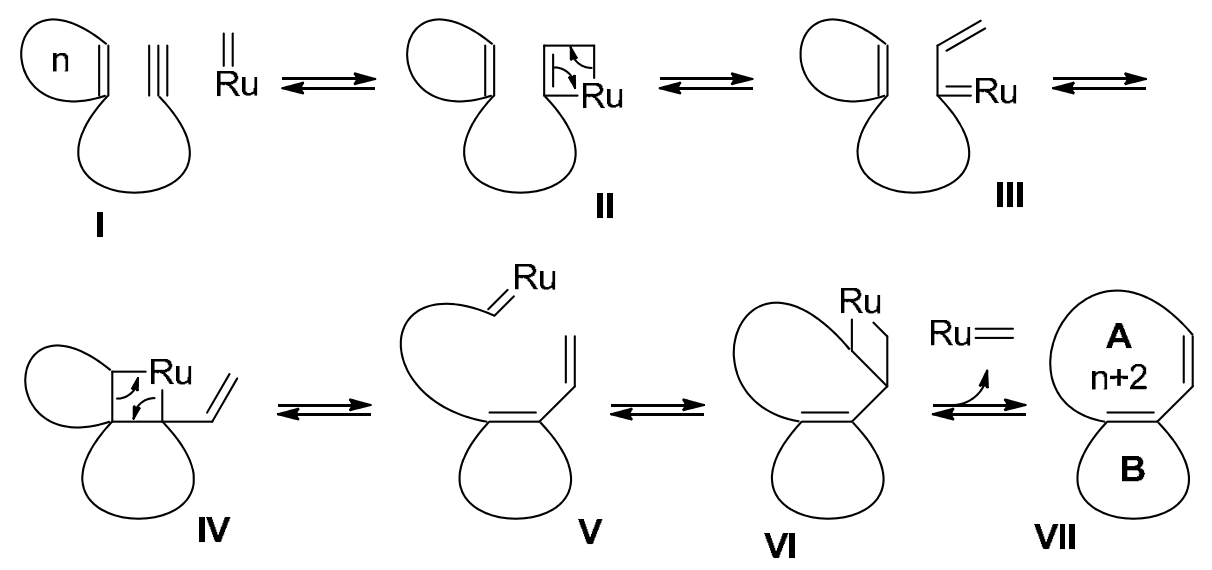

Scheme 3. Plausible reaction mechanism for ROM-RCM of cycloalkene-ynes. 
The reaction of the alkyne part of enyne $\mathbf{I}$ with a ruthenium methylidene complex gives ruthenacyclobutene II, which was converted into vinyl carbene complex III. If this carbene complex III reacts with a cycloalkene part, ruthenacyclobutane IV would be formed and it should be converted into $\mathbf{V}$. If carbene complex $\mathbf{V}$ reacts with a vinyl group intramolecularly, ring-closing metathesis would proceed to provide bicyclic compound VII, together with a ruthenium methylidene complex. Interestingly, the initial $n$-membered ring is converted into an $(n+2)$-membered ring size in this reaction. The other ring size depends on the chain lengths between the double bond and the triple bond. We recently developed ROM-RCM of cyclobutenylmethylamine $\mathbf{4}$ having an alkyne moiety in a tether catalyzed by second-generation Grubbs catalyst 1, and isoquinoline derivatives 5 could be obtained in good yields in a one-step reaction (Scheme 4). ${ }^{4 \mathrm{~d}}$
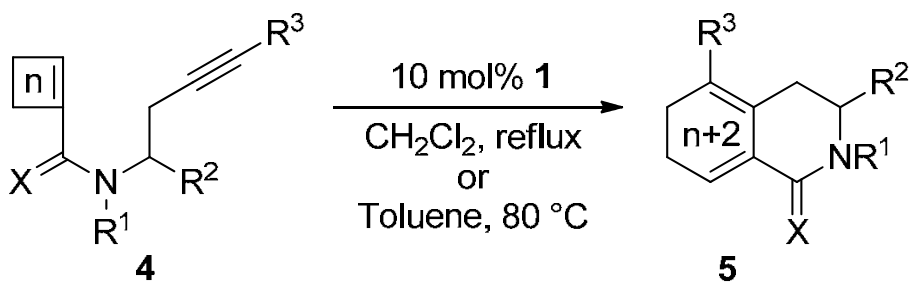

Scheme 4. ROM-RCM of cyclobutene-ynes.

Herein we report the synthesis of tricyclic compounds 7 by ROM-RCM of bicyclo[4.2.0]octene-ynes $\mathbf{6}$ for the further application of our previous development (Scheme 5).

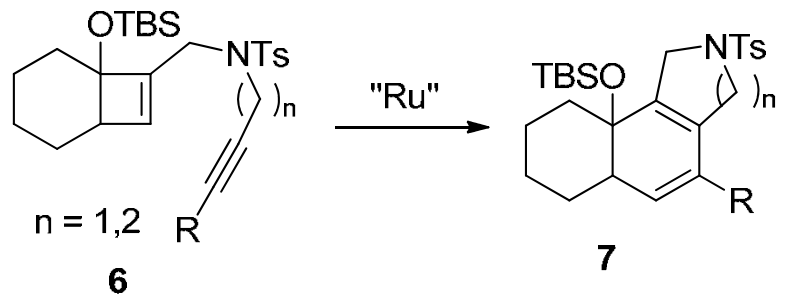

Scheme 5. Plan for synthesis of tricyclic heterocycles.

\section{Results and Discussion}

Enynes 6 were prepared according to the synthetic route shown in Scheme 6. Alcohol 9 was synthesized by the literature procedure. ${ }^{6}[2+2]$ cocyclization of silyl enol ether $\mathbf{8}$ and ethyl propiolate, promoted by $\mathrm{ZrCl}_{4}$ and followed by treatment with DIBAL-H, gave primary alcohol $\mathbf{9}$ in 66\% yield. Enynes 6a c were obtained by a Mitsunobu reaction ${ }^{7}$ of $\mathbf{9}$ with tosylamide 10 or 
11 in good to high yield. Enyne 6d, having a phenyl group on the terminal alkyne, was synthesized by Sonogashira cross coupling ${ }^{8}$ of $\mathbf{6} \mathbf{b}$ and Iodobenzene.

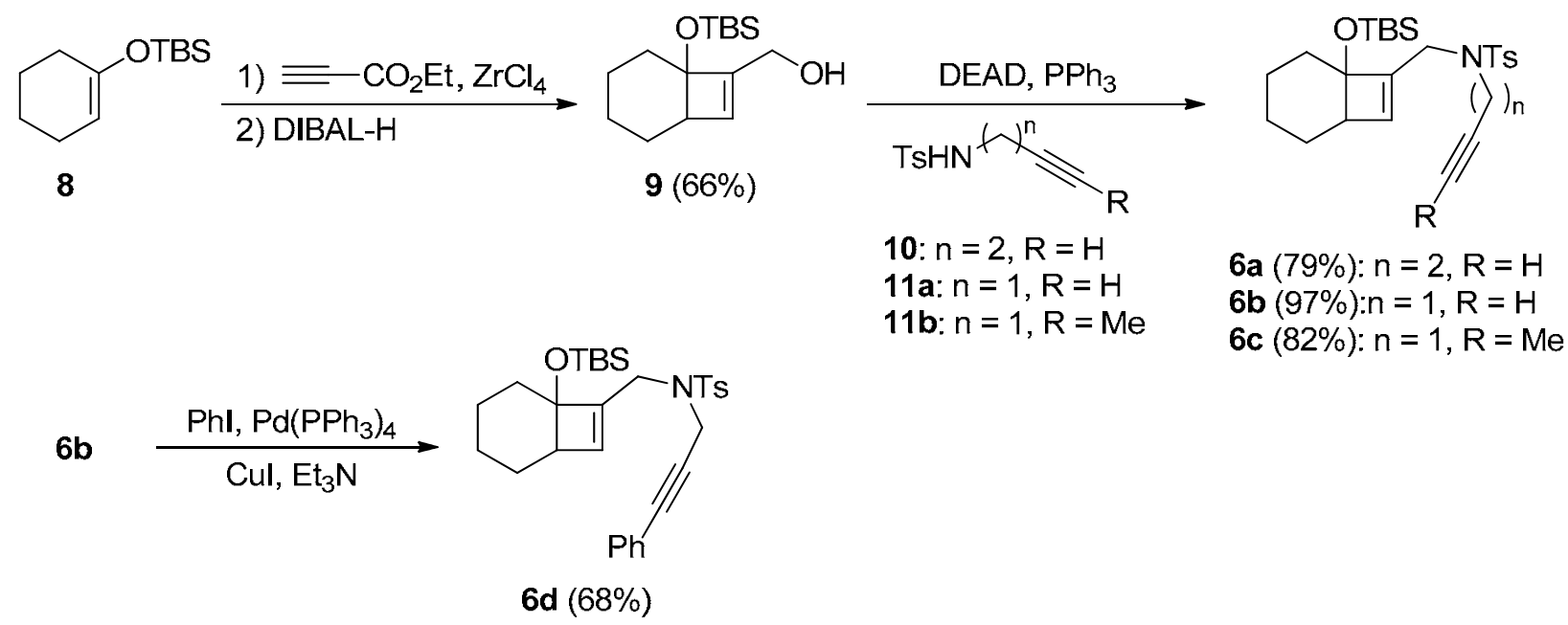

Scheme 6. Preparation of substrate 6.

ROM-RCM of $\mathbf{6 a}$ was examined and the results are shown in Table 1 . When a reaction of $\mathbf{6 a}$ was carried out in the presence of $20 \mathrm{~mol} \%$ of ruthenium carbene complex 1 in $\mathrm{CH}_{2} \mathrm{Cl}_{2}$ under an ethylene atmosphere and reflux for 48 h, compound 12 was obtained in 24\% yield (entry 1). Although tricyclic compound 7a was not obtained, changing a solvent from $\mathrm{CH}_{2} \mathrm{Cl}_{2}$ to toluene was effective for the synthesis of $\mathbf{1 2}$ and conjugated diene derivative $\mathbf{1 2}$ was obtained in $90 \%$ yield (entry 2).

Table 1. ROM-RCM of $6 \mathbf{a}^{\mathrm{a}}$<smiles>C#CCC[NH2+]CC1=CC2CCCCC12O</smiles><smiles>C=CC(C)C</smiles><smiles>C=CC(=C)CC[NH+]([S-])CC1=CC2CCCCC12O</smiles>

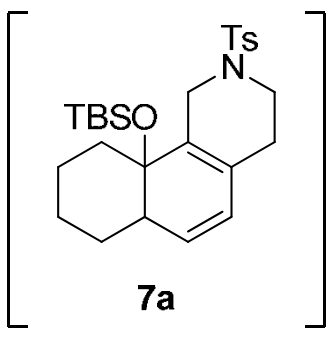

\begin{tabular}{cccc}
\hline Entry & Solvent & Time (h) & Yield of 12 (\%) \\
\hline 1 & $\mathrm{CH}_{2} \mathrm{Cl}_{2}$ & 48 & 24 \\
2 & Toluene & 0.5 & 90 \\
\hline
\end{tabular}

${ }^{\mathrm{a}}$ All reactions were carried out in the presence of $20 \mathrm{~mol} \% \mathbf{1}$ under ethylene atmosphere. 
Probably, ruthenium carbene complex $\mathbf{1}$ would react with the alkyne part of $\mathbf{6 a}$ to provide carbene complex ii, although a recent study strongly supported the predominance of the initial cyclometallation on an alkene over an alkyne. ${ }^{9}$ Ruthenium carbene complex ii reacts with ethylene intermolecularly, not the alkene part of cycloalkene. Then triene derivative $\mathbf{1 2}$ would be obtained through ruthenacyclobutane intermediate iii (Scheme 7, path a).

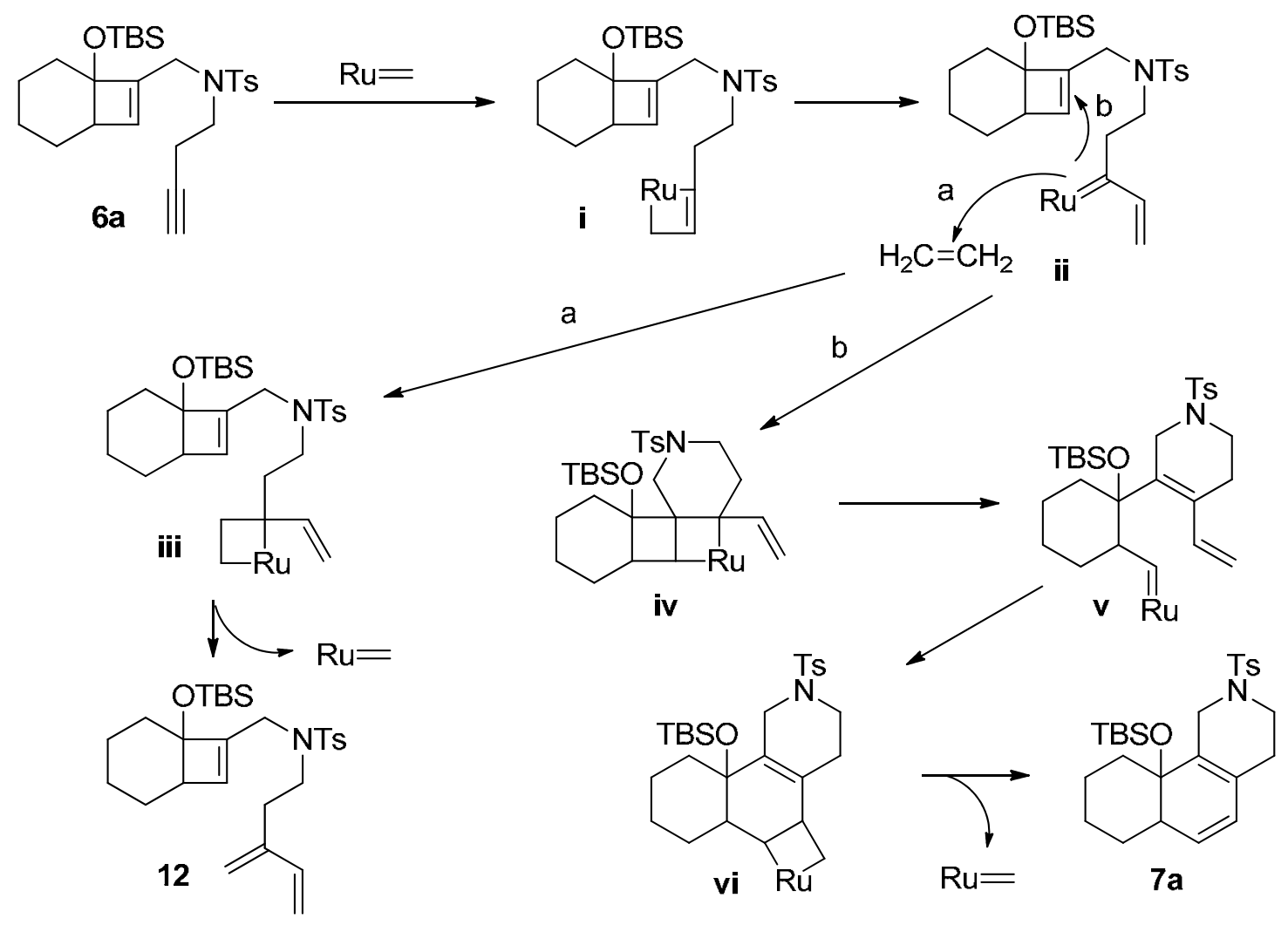

Scheme 7. Possible reaction pathway.

Enyne 6b, which has a retrenched alkyne side chain compared to 6a, was examined (Scheme 8). When a dichloromethane solution of $\mathbf{6 b}$ was stirred in the presence of $10 \mathrm{~mol} \%$ of $\mathbf{1}$ under reflux for $1 \mathrm{~h}$, desilylated tricyclic compound $\mathbf{1 3}$ was isolated in $60 \%$ yield after the usual workup by column chromatography on silica gel. The crude product was explored by ${ }^{1} \mathrm{H}$ NMR measurement in order to get to the root of the desilylation. As a result, we could confirm that non-aromatized product $\mathbf{7 b}$ was obtained in $69 \%$ yield. Presumably, ROM-RCM of $\mathbf{6 b}$ proceeded to provide $\mathbf{7 b}$, which was aromatized to $\mathbf{1 3}$ during column chromatography on silica gel. 


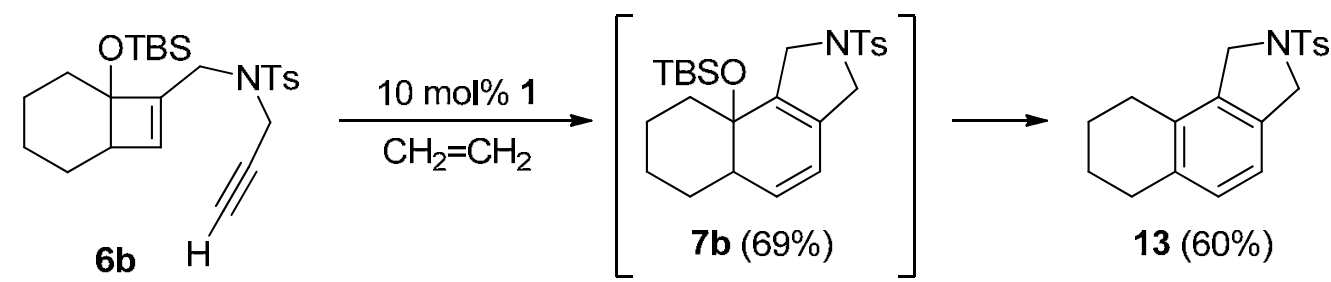

Scheme 8. ROM-RCM of $\mathbf{6 b}$.

Subsequently, the effect of the substituent on the terminal alkyne was examined (Table 2). When a $\mathrm{CH}_{2} \mathrm{Cl}_{2}$ solution of $\mathbf{6 c}$ having a methyl group on the alkyne was stirred in the presence of 10 mol\% of 1 under reflux for 1 h, triene derivative 14c was obtained in 72\% yield (entry 1). Although we found that the reaction of phenyl-substituted ene-ynamide $\mathbf{6 d}$ did not proceed to recover $\mathbf{6 d}$ in $79 \%$ yield (entry 2 ), triene derivative 14d could be obtained in $98 \%$ yield after the stirring of $6 \mathbf{d}$ at $80{ }^{\circ} \mathrm{C}$ for $0.5 \mathrm{~h}$ in toluene (entry 3).

Table 2. Substituent effect on the alkynes
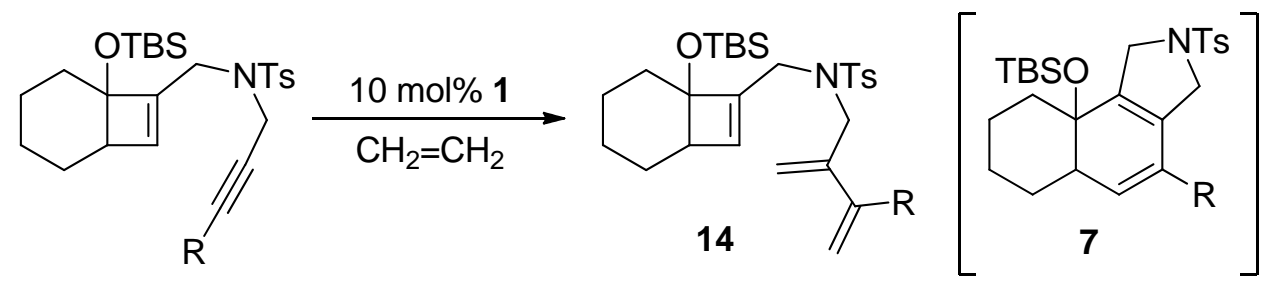

6c: $\mathrm{R}=\mathrm{Me}$

6d: $\mathrm{R}=\mathrm{Ph}$

\begin{tabular}{cccccccc}
\hline Entry & $\mathrm{R}$ & Solvent & $\begin{array}{c}\text { Temp. } \\
\left({ }^{\circ} \mathrm{C}\right)\end{array}$ & $\begin{array}{c}\text { Time } \\
(\mathrm{h})\end{array}$ & $\begin{array}{c}\text { Yield of } \mathbf{1 4} \\
(\%)\end{array}$ & $\begin{array}{c}\text { Yield of } \mathbf{7} \\
(\%)\end{array}$ & $\begin{array}{c}\text { Recovery of } \mathbf{6} \\
(\%)\end{array}$ \\
\hline 1 & $\mathrm{Me}$ & $\mathrm{CH}_{2} \mathrm{Cl}_{2}$ & Reflux & 1 & 72 & 0 & 0 \\
2 & $\mathrm{Ph}$ & $\mathrm{CH}_{2} \mathrm{Cl}_{2}$ & Reflux & 24 & 0 & 0 & 79 \\
3 & $\mathrm{Ph}$ & Toluene & 80 & 0.5 & 98 & 0 & 0 \\
\hline
\end{tabular}

\section{Conclusions}

We have studied the ROM-RCM of bicyclo[4.2.0]octene-ynes 6. When enyne 6a was used as the substrate, intermolecular CM with ethylene proceeded to give triene derivative 12. The improved yield of 12 was confirmed not so much in the case of $\mathrm{CH}_{2} \mathrm{Cl}_{2}$ but as toluene. ROM-RCM of cycloalkene-yne $\mathbf{6 b}$ proceeded smoothly to provide tricyclic compound $\mathbf{7 b}$, which aromatized easily to 6,6,5-fused ring compound $\mathbf{1 3}$ by desilylation under purification on column chromatography. The intermolecular CM of $\mathbf{6 c , d}$ with ethylene proceeded to give triene 
derivative 14 in high yield although remarkable advancement was not observed when the substituent on the alkyne was examined.

\section{Experimental Section}

General. The metathesis reactions were carried out under an atmosphere of ethylene (1 atm) unless otherwise mentioned. All other manipulations were carried out under an atmosphere of argon unless otherwise mentioned. Ruthenium complexes were purchased from Aldrich Chemical Company. All other solvents and reagents were purified when necessary using standard procedure. Column chromatography was performed on silica gel $60 \mathrm{~N}$ (spherical, neutral, 40-60 $\mu \mathrm{m}$, Kanto Chemical Co.). IR spectra were recorded on PERKIN ELMER FT-IR 1725X. ${ }^{1} \mathrm{H}$ and ${ }^{13} \mathrm{C}$ NMR spectra were recorded on JEOL JNM-EX270 $\left({ }^{1} \mathrm{H}: 270 \mathrm{MHz},{ }^{13} \mathrm{C}: 67.8\right.$ $\mathrm{MHz}$ ) spectrometer. Chemical shift values were reported in ppm $(\delta)$ downfield from tetramethylsilane as an internal standard, or residual solvent peak $\left[{ }^{1} \mathrm{H} \mathrm{NMR}, \mathrm{CHCl}_{3}(7.24):{ }^{13} \mathrm{C}\right.$ NMR, $\mathrm{CHCl}_{3}$ (77.0)]. Coupling constants $(J)$ are reported in Hertz (Hz). EI mass spectra were measured on JEOL JMN-DX 303/JMA-DA 5000.

\section{General procedure A for metathesis reaction}

To a solution of cycloalkene-ynes in $\mathrm{CH}_{2} \mathrm{Cl}_{2}(0.02 \mathrm{M})$ was added ruthenium carbene complex $\mathbf{1}$, and the mixture was refluxed under ethylene atmosphere. Ethylvinyl ether was added to the mixture at $0{ }^{\circ} \mathrm{C}$, and the volatiles were removed under reduce pressure. The residue was purified by column chromatography on silica gel to provide the product.

\section{General procedure $B$ for metathesis reaction}

To a solution of cycloalkene-ynes in toluene $(0.02 \mathrm{M})$ was added ruthenium carbene complex $\mathbf{1}$, and the mixture was stirred at $80{ }^{\circ} \mathrm{C}$ under ethylene atmosphere. Ethylvinyl ether was added to the mixture at $0{ }^{\circ} \mathrm{C}$, and the volatiles were removed under reduce pressure. The residue was purified by column chromatography on silica gel to provide the product.

[6-(t-Butyldimethylsilyloxy)-bicyclo[4.2.0]oct-7-en-7-yl]-methanol (9). To a suspension of $\mathrm{ZrCl}_{4}(1.34 \mathrm{~g}, 5.73 \mathrm{mmol})$ in $\mathrm{CH}_{2} \mathrm{Cl}_{2}(20 \mathrm{~mL})$ was added a solution of 8 (1.22 g, $\left.5.73 \mathrm{mmol}\right)$ in $\mathrm{CH}_{2} \mathrm{Cl}_{2}(9 \mathrm{~mL})$ after the addition of Ethyl propiolate $(0.87 \mathrm{~mL}, 8.60 \mathrm{mmol})$ at $-78{ }^{\circ} \mathrm{C}$, and the reaction mixture was stirred for $20 \mathrm{~min}$. The reaction mixture was warmed to room temperature after addition of Diethyl ether $(30 \mathrm{~mL})$ and $\mathrm{H}_{2} \mathrm{O}(10 \mathrm{~mL})$ at $-78{ }^{\circ} \mathrm{C}$, and water phase was separated. The organic phase was washed with saturated $\mathrm{NaCl}$ solution, and dried with $\mathrm{MgSO}_{4}$. The volatiles were removed under reduce pressure to obtain crude product, which was used directly next reaction. To a solution of the crude product in THF (4.8 mL) was added DIBAL-H (2.9 mL, $2.9 \mathrm{mmol}, 1 \mathrm{M}$ THF solution) at $-78{ }^{\circ} \mathrm{C}$, and the mixture was stirred for $1 \mathrm{~h}$ at the same temperature. Ethyl acetate $(6 \mathrm{~mL})$ and saturated potassium sodium tartrate solution $(30 \mathrm{~mL})$ was added to the mixture, which was warmed to room temperature and stirred over $12 \mathrm{~h}$. The mixture 
was extracted with ethyl acetate. The organic phase was washed with saturated $\mathrm{NaCl}$ solution, and dried with $\mathrm{MgSO}_{4}$. The volatiles were removed under reduce pressure, and the residue was purified by column chromatography on silica gel (Hexane / EtOAc, 3:1) to provide 9 (169.3 mg, 66\%); ${ }^{1} \mathrm{H}$ NMR (270 MHz, $\left.\mathrm{CDCl}_{3}\right) \delta 0.09$ (s, 3H), 0.10 (s, 3H), 0.86 (s, 9H), 1.39-1.60 (m, 4H), 1.66-1.85 (m, 4H), 2.70-2.72 (m, 1H), 4.14-4.24 (m, 2H), 5.98 (dd, $J=1.5,2.5 \mathrm{~Hz}, 1 \mathrm{H})$.

$N$-[6-(t-Butyldimethylsilyloxy)-bicyclo[4.2.0]oct-7-en-7-ylmethyl]- $N$-but-3-ynyl-p-

toluenesulfonamide (6a). To a solution of 9 (325.7 mg, $1.21 \mathrm{mmol}), 10$ (379.6 mg, $1.70 \mathrm{mmol}$ ) and $\mathrm{PPh}_{3}$ (634.7 mg, $\left.2.42 \mathrm{mmol}\right)$ in THF (12 mL, 0.1 M) was added DEAD (1.1 mL, $\left.2.42 \mathrm{mmol}\right)$ at $0{ }^{\circ} \mathrm{C}$, and the mixture was stirred at room temperature for $22 \mathrm{~h}$. The volatiles were removed under reduce pressure, and the residue was purified by column chromatography on silica gel (Hexane / $\mathrm{Et}_{2} \mathrm{O}, 5: 1$ ) to provide 6a (453.8 mg, 79\%); IR (neat) v 3312, 2930, 2856, 2122, 1600, 1346, 1254, 1161, $1100 \mathrm{~cm}^{-1}$; ${ }^{1} \mathrm{H}$ NMR (270 MHz, $\mathrm{CDCl}_{3}$ ) $\delta 0.06$ (s, 3H), 0.07 (s, 3H), 0.85 (s, $9 \mathrm{H}), 1.26-1.69(\mathrm{~m}, 8 \mathrm{H}), 1.95(\mathrm{t}, J=2.6 \mathrm{~Hz}, 1 \mathrm{H}), 2.42-2.51(\mathrm{~m}, 5 \mathrm{H}), 2.59(\mathrm{~s}, 1 \mathrm{H}), 3.42$ (tt, $J=$ 1.8, $7.8 \mathrm{~Hz}, 2 \mathrm{H}), 3.78$ (dt, $J=18.0,1.8 \mathrm{~Hz}, 1 \mathrm{H}), 3.92$ (dt, $J=18.0,1.8 \mathrm{~Hz}, 1 \mathrm{H}), 5.58(\mathrm{~d}, J=0.8$ $\mathrm{Hz}, 1 \mathrm{H}), 7.28$ (d, $J=8.4 \mathrm{~Hz}, 2 \mathrm{H}), 7.72(\mathrm{~d}, J=8.4 \mathrm{~Hz}, 2 \mathrm{H}) ;{ }^{13} \mathrm{C}$ NMR $\left(67.8 \mathrm{MHz}, \mathrm{CDCl}_{3}\right) \delta-2.8$, -2.6, 17.6, 18.0, 18.2, 19.3, 21.7, 23.2, 25.9, 32.1, 44.3, 46.6, 49.0, 70.3, 78.4, 81.2, 127.4, 129.8, 132.9, 137.4, 143.5, 147.7; EI-LRMS m/z $473\left(\mathrm{M}^{+}\right)$, 416, 280, 91, 73; EI-HRMS m/z calcd for $\mathrm{C}_{26} \mathrm{H}_{39} \mathrm{O}_{3} \mathrm{NSiS}\left(\mathrm{M}^{+}\right)$473.2420, found 473.2430.

$N$-[6-(t-Butyldimethylsilyloxy)-bicyclo[4.2.0]oct-7-en-7-ylmethyl]- $N$-prop-2-ynyl-p-

toluenesulfonamide (6b). To a solution of 9 (206.3 mg, $0.77 \mathrm{mmol})$, 11a (225.1 $\mathrm{mg}, 1.08 \mathrm{mmol})$ and $\mathrm{PPh}_{3}$ (403.1 mg, $\left.1.54 \mathrm{mmol}\right)$ in THF (7 mL, 0.1 M) was added DEAD (0.7 mL, $\left.1.54 \mathrm{mmol}\right)$ at $0{ }^{\circ} \mathrm{C}$, and the mixture was stirred at room temperature for $24 \mathrm{~h}$. The volatiles were removed under reduce pressure, and the residue was purified by column chromatography on silica gel (Hexane / $\mathrm{Et}_{2} \mathrm{O}, 5: 1$ ) to provide $6 \mathbf{b}$ (346.1 mg, 97\%); IR (neat) v 3248, 2927, 2854, 2116, 1599, 1353, 1253, 1186, $1095 \mathrm{~cm}^{-1}$; ${ }^{1} \mathrm{H}$ NMR (270 MHz, $\mathrm{CDCl}_{3}$ ) $\delta 0.07$ (s, 3H), 0.08 (s, 3H), 0.85 (s, 9H), 1.41-1.55 (m, 8H), 2.00 (t, $J=2.4 \mathrm{~Hz}, 1 \mathrm{H}), 2.42$ (s, 3H), 2.67 (s, 1H), $3.81(\mathrm{~s}, 2 \mathrm{H}), 4.23$ (t, $J=2.6 \mathrm{~Hz}, 2 \mathrm{H}), 5.95$ (s, 1H), 7.28 (d, $J=8.4 \mathrm{~Hz}, 2 \mathrm{H}), 7.74(\mathrm{~d}, J=8.4 \mathrm{~Hz}, 2 \mathrm{H}) ;{ }^{13} \mathrm{C}$ NMR $(67.8$ $\left.\mathrm{MHz} \mathrm{CDCl}_{3}\right) \delta-3.3,-3.1,17.1,17.5,17.7,21.2,22.7,25.4,31.6,36.1,42.2,48.7,73.5,76.4$, 79.2, 127.4, 129.1, 133.6, 135.9, 143.1, 146.6; EI-LRMS m/z 459(M+1), 402, 266, 91, 73; EIHRMS $m / z$ calcd for $\mathrm{C}_{25} \mathrm{H}_{37} \mathrm{O}_{3} \mathrm{NSiS}\left(\mathrm{M}^{+}\right)$459.2263, found 459.2255.

\section{$N$-[6-(t-Butyldimethylsilyloxy)-bicyclo[4.2.0]oct-7-en-7-ylmethyl]- $N$-but-2-ynyl-p-}

toluenesulfonamide (6c). To a solution of 9 (169.3 mg, $0.63 \mathrm{mmol}), \mathbf{1 1 b}(198.2 \mathrm{mg}, 0.43 \mathrm{mmol})$ and $\mathrm{PPh}_{3}$ (344.2 mg, $0.43 \mathrm{mmol}$ ) in THF (4 mL, 0.1 M) was added DEAD (0.6 mL, $0.43 \mathrm{mmol}$ ) at $0{ }^{\circ} \mathrm{C}$, and the mixture was stirred at room temperature for $48 \mathrm{~h}$. The volatiles were removed under reduce pressure, and the residue was purified by column chromatography on silica gel (Hexane / $\left.\mathrm{Et}_{2} \mathrm{O}, 4: 1\right)$ to provide 6c (245.6 mg, 82\%); IR (neat) v 2929, 2856, 2225, 1600, 1352, 1254, 1163, $1095 \mathrm{~cm}^{-1}$; ${ }^{1} \mathrm{H}$ NMR (270 MHz, $\left.\mathrm{CDCl}_{3}\right) \delta 0.07$ (s, 3H), 0.08 (s, 3H), 0.85 (s, 9H), 1.39-1.56 (m, 11H), 2.42 (s, 3H), 2.66 (s, 1H), 3.78 (t, $J=2.0 \mathrm{~Hz}, 2 \mathrm{H}), 4.14$ (tq, $J=2.0,2.3 \mathrm{~Hz}$, 2H), 5.92 (d, $J=0.8 \mathrm{~Hz}, 1 \mathrm{H}), 7.28$ (d, $J=8.1 \mathrm{~Hz}, 2 \mathrm{H}), 7.74$ (d, $J=8.1 \mathrm{~Hz}, 2 \mathrm{H}) ;{ }^{13} \mathrm{C}$ NMR $(67.8$ $\left.\mathrm{MHz}, \mathrm{CDCl}_{3}\right) \delta$-3.2, -2.9, 3.1, 17.4, 17.7, 17.9, 21.4, 23.0, 25.6, 31.9, 36.9, 42.4, 48.9, 71.8, 
78.3, 81.5, 127.8, 129.1, 133.2, 136.3, 143.0, 147.2; EI-LRMS m/z $473\left(\mathrm{M}^{+}\right), 416,280,91,73$; EI-HRMS $m / z$ cacld for $\mathrm{C}_{26} \mathrm{H}_{39} \mathrm{O}_{3} \mathrm{NSiS}\left(\mathrm{M}^{+}\right)$473.2420, found 473.2420.

$N$-[6-(t-Butyldimethylsilyloxy)-bicyclo[4.2.0]oct-7-en-7-ylmethyl]- $N$-(3-phenyl-prop-2-ynyl)p-toluenesulfonamide (6d). To a solution of $\mathrm{Pd}\left(\mathrm{PPh}_{3}\right)_{4}(25.1 \mathrm{mg}, 0.02 \mathrm{mmol})$ and $\mathrm{CuI}$ (4.1 mg, $0.02 \mathrm{mmol})$ in $\mathrm{Et}_{3} \mathrm{~N}(1 \mathrm{~mL}, 0.4 \mathrm{M})$ was added $\mathrm{PhI}(51 \mu \mathrm{L}, 0.46 \mathrm{mmol})$ at room temperature, and the mixture was stirred for $10 \mathrm{~min}$. To the mixture was added $\mathbf{6 b}$ ( $200.4 \mathrm{mg}, 0.43 \mathrm{mmol}$ ) in Benzene $(1 \mathrm{~mL})$, and the resulting mixture was stirred at the same temperature for $19 \mathrm{~h}$. The volatiles were removed under reduce pressure, and the residue was purified by column chromatography on silica gel (Hexane / $\mathrm{Et}_{2} \mathrm{O}, 5: 1$ ) to provide 6d (156.8 mg, 68\%); IR (neat) $\mathrm{v}$ 2929, 2856, 2242, 1599, 1352, 1254, 1164, $1094 \mathrm{~cm}^{-1} ;{ }^{1} \mathrm{H}$ NMR (270 MHz, $\left.\mathrm{CDCl}_{3}\right) \delta 0.08$ (s, 3H), 0,09 (s, 3H), 0.85 (s, 9H), 1.43-1.54 (m, 8H), 2.33 (s, 3H), 2.70 (s, 1H), 3.87 (d, J = 1.8 Hz, 2H), 4.44 (d, $J=2.3 \mathrm{~Hz}, 2 \mathrm{H}), 6.03$ (d, $J=1.0 \mathrm{~Hz}, 1 \mathrm{H}), 7.05$ (d, $J=8.3 \mathrm{~Hz}, 2 \mathrm{H}), 7.20-7.28$ (m, 5H), 7.77 (d, $J=8.3 \mathrm{~Hz}, 2 \mathrm{H}) ;{ }^{13} \mathrm{C}$ NMR $\left(67.8 \mathrm{MHz}, \mathrm{CDCl}_{3}\right) \delta-3.1,-2.8,17.4,17.8,17.9,21.3$, 23.0, 25.7, 31.9, 37.3, 42.8, 49.1, 78.5, 81.9, 85.7, 122.2, 127.7, 128.0, 128.2, 129.4, 131.4, 133.8, 136.1, 143.3, 147.0; EI-LRMS m/z $536\left(\mathrm{M}^{+}\right)$, 478, 380, 342, 248, 115, 91, 73; EI-HRMS $\mathrm{m} / \mathrm{z}$ cacld for $\mathrm{C}_{31} \mathrm{H}_{41} \mathrm{O}_{3} \mathrm{NSiS}\left(\mathrm{M}^{+}\right)$535.2576, found 535.2576.

$N$-[6-(t-Butyldimethylsilyloxy)-bicyclo[4.2.0]oct-7-en-7-ylmethyl]- $N$-(3-methylene-pent-4enyl)-p-toluenesulfonamide (12). According to general procedure B, a solution of 6 a (33.0 mg, $0.07 \mathrm{mmol}$ ) and 1 (5.9 mg, $7 \mu \mathrm{mol})$ in toluene $(3.5 \mathrm{~mL}, 0.02 \mathrm{M})$ was stirred for $0.5 \mathrm{~h}$ to provide 12 (31.6 mg, 90\%); IR (neat) $v$ 2930, 2856, 1597, 1345, 1254, 1160, $1100 \mathrm{~cm}^{-1}$; ${ }^{1} \mathrm{H}$ NMR $(270$ $\left.\mathrm{MHz} \mathrm{CDCl}_{3}\right) \delta 0.04$ (s, 3H), 0.06 (s, 3H), 0.84 (s, 9H), 1.26-1.52 (m, 8H), 2.41 (s, 3H), 2.492.58 (m, 3H), 3.35 (tt, $J=2.1,6.5 \mathrm{~Hz}, 2 \mathrm{H}), 3.78$ (dt, $J=18.1,2.1 \mathrm{~Hz}, 1 \mathrm{H}), 3.89$ (dt, $J=18.1,2.1$ Hz, 1H), 4.99 (s, 1H), 5.04 (s, 1H), 5.08 (d, $J=10.9 \mathrm{~Hz}, 1 \mathrm{H}), 5.28$ (d, $J=17.6 \mathrm{~Hz}, 1 \mathrm{H}), 5.56$ (s, 1H), 6.31 (dd, $J=10.9,17.6 \mathrm{~Hz}, 1 \mathrm{H}), 7.27$ (d, $J=8.2 \mathrm{~Hz}, 2 \mathrm{H}), 7.71(\mathrm{~d}, J=8.2 \mathrm{~Hz}, 2 \mathrm{H}) ;{ }^{13} \mathrm{C}$ NMR (67.8 MHz, $\mathrm{CDCl}_{3}$ ) $\delta$-3.1, -2.8, 17.3, 17.8, 17.9, 21.4, 23.0, 25.6, 31.0, 31.9, 43.9, 47.2, 48.7, 78.1, 114.0, 117.5, 127.2, 129.5, 132.2, 137.5, 138.0, 143.0, 143.1, 147.7; EI-LRMS m/z 501 $\left(\mathrm{M}^{+}\right)$, 444, 346, 308, 251, 91, 73; EI-HRMS $\mathrm{m} / \mathrm{z}$ cacld for $\mathrm{C}_{28} \mathrm{H}_{43} \mathrm{O}_{3} \mathrm{NSiS}\left(\mathrm{M}^{+}\right)$501.2733, found 501.2732.

2-(p-Toluenesulfonyl)-2,3,6,7,8,9-hexahydro-1H-benzo[e]isoindole (13). According to general procedure A, a solution of $\mathbf{6 b}(57.2 \mathrm{mg}, 0.12 \mathrm{mmol})$ and $\mathbf{1}$ (10.5 mg, $12.4 \mu \mathrm{mol})$ in $\mathrm{CH}_{2} \mathrm{Cl}_{2}$ (6 $\mathrm{mL}, 0.02 \mathrm{M}$ ) was stirred for $1 \mathrm{~h}$ to provide 13 (23.1 mg, 60\%); IR (neat) $v$ 2936, 1596, 1345, $1164 \mathrm{~cm}^{-1} ;{ }^{1} \mathrm{H}$ NMR (270 MHz, $\left.\mathrm{CDCl}_{3}\right) \delta 1.77(\mathrm{t}, J=3.2 \mathrm{~Hz}, 4 \mathrm{H}), 2.40(\mathrm{~s}, 3 \mathrm{H}), 2.52(\mathrm{t}, J=5.6$ $\mathrm{Hz}, 2 \mathrm{H}), 2.72$ (t, $J=5.4 \mathrm{~Hz}, 2 \mathrm{H}), 4.50(\mathrm{~s}, 2 \mathrm{H}), 4.60$ (s, 2H), 6.89 (d, $J=7.9 \mathrm{~Hz}, 1 \mathrm{H}), 6.96(\mathrm{~d}, J=$ $7.8 \mathrm{~Hz}, 1 \mathrm{H}), 7.31$ (d, $J=8.2 \mathrm{~Hz}, 2 \mathrm{H}), 7.77$ (d, $J=8.2 \mathrm{~Hz}, 2 \mathrm{H}) ;{ }^{13} \mathrm{C}$ NMR $\left(67.8 \mathrm{MHz}, \mathrm{CDCl}_{3}\right) \delta$ 21.4, 22.6, 22.8, 26.2, 29.2, 52.9, 53.9, 119.3, 127.5, 128.8, 129.7, 131.9, 132.8, 133.8, 134.9, 136.5, 143.5; EI-LRMS m/z 326(M $\left(\mathrm{M}^{+}\right)$, 172, 91; EI-HRMS $\mathrm{m} / \mathrm{z}$ cacld for $\mathrm{C}_{19} \mathrm{H}_{21} \mathrm{O}_{2} \mathrm{NS}_{\left(\mathrm{M}^{+}\right)}$ 327.1293, found 327.1274.

$N$-[6-(t-Butyldimethylsilyloxy)-bicyclo[4.2.0]oct-7-en-7-ylmethyl]- $N$-(3-methyl-2-methylenebut-3-enyl)-p-toluenesulfonamide (14c). According to general procedure A, a solution of $\mathbf{6 c}$ (63.1 mg, $0.13 \mathrm{mmol})$ and 1 (11.3 mg, $13.33 \mu \mathrm{mol})$ in $\mathrm{CH}_{2} \mathrm{Cl}_{2}(6.5 \mathrm{~mL}, 0.02 \mathrm{M})$ was stirred for 1 
h to provide 14c (47.1 mg, 72\%); IR (neat) v 2930, 2856, 1345, 1254, 1162, $1096 \mathrm{~cm}^{-1} ;{ }^{1} \mathrm{H} \mathrm{NMR}$ $\left(270 \mathrm{MHz}, \mathrm{CDCl}_{3}\right) \delta 0.02$ (s, 3H), 0.03 (s, 3H), 0.82 (s, 9H), 1.26-1.45 (m, 8H), 1.89 (s, 3H), 2.41 (s, 3H), 2.50 (s, 1H), 3.75 (d, $J=2.1 \mathrm{~Hz}, 2 \mathrm{H}), 4.14$ (q, $J=15.0 \mathrm{~Hz}, 2 \mathrm{H}), 5.02$ (s, 1H), 5.14 (s, 1H), 5.23 (s, 2H), 5.29 (s, 1H), 7.27 (d, $J=8.2 \mathrm{~Hz}, 2 \mathrm{H}), 7.73$ (d, $J=8.2 \mathrm{~Hz}, 2 \mathrm{H}) ;{ }^{13} \mathrm{C}$ NMR $\left(67.8 \mathrm{MHz}, \mathrm{CDCl}_{3}\right) \delta$-3.1, -2.9, 17.4, 17.7, 17.9, 21.2, 21.4, 23.0, 25.6, 31.9, 42.9, 48.7, 49.9, 78.0, 114.0, 115.4, 127.4, 129.4, 131.5, 137.4, 140.6, 141.4, 143.0, 147.4; EI-LRMS m/z 501 $\left(\mathrm{M}^{+}\right)$, 444, 362, 346, 308, 91, 73; EI-HRMS m/z calcd for $\mathrm{C}_{28} \mathrm{H}_{43} \mathrm{O}_{3} \mathrm{NSiS}\left(\mathrm{M}^{+}\right)$501.2733, found 501.2740 .

$N$-[6-(t-Butyldimethylsilyloxy)-bicyclo[4.2.0]oct-7-en-7-ylmethyl]- $N$-(2-methylene-3-phenylbut-3-enyl)-p-toluenesulfonamide (14d). According to general procedure $B$, a solution of $\mathbf{6 d}$ (29.4 mg, $0.05 \mathrm{mmol}$ ) and 1 (4.6 mg, $5.47 \mu \mathrm{mol}$ ) in toluene (3 mL, $0.02 \mathrm{M}$ ) was stirred for $0.5 \mathrm{~h}$ to provide 14d (27.8 mg, 98\%); IR (neat) $v$ 2930, 2856, 1599, 1344, 1256, 1160, $1094 \mathrm{~cm}^{-1} ;{ }^{1} \mathrm{H}$ NMR (270 MHz, $\left.\mathrm{CDCl}_{3}\right) \delta 0.03$ (s, 3H), 0.05 (s, 3H), 0.84 (s, 9H), 1.26-1.46 (m, 8H), 2.40 (s, 3H), 2.53 (s, 1H), 3.85 (s, 2H), 4.16 (s, 2H), 5.13 (s, 1H), 5.23 (s, 1H), 5.30 (s, 1H), 5.35 (d, $J=$ $0.8 \mathrm{~Hz}, 1 \mathrm{H}), 5.38$ (d, $J=1.0 \mathrm{~Hz}, 1 \mathrm{H}), 7.24-7.34(\mathrm{~m}, 7 \mathrm{H}), 7.72(\mathrm{~d}, J=8.4 \mathrm{~Hz}, 2 \mathrm{H}) ;{ }^{13} \mathrm{C}$ NMR $\left(67.8 \mathrm{MHz}, \mathrm{CDCl}_{3}\right) \delta$-3.0, -2.8, 17.4, 17.8, 18.0, 21.5, 23.0, 25.7, 31.9, 43.1, 48.7, 50.2, 78.1, 115.1, 118.6, 127.4, 127.5, 128.1, 128.1, 129.4, 131.9, 137.6, 140.6, 142.4, 143.0, 147.3, 148.0; EI-LRMS m/z $564\left(\mathrm{M}^{+}\right)$, 507, 408, 370, 251, 91, 73; EI-HRMS m/z cacld for $\mathrm{C}_{33} \mathrm{H}_{45} \mathrm{O}_{3} \mathrm{NSiS}_{\left(\mathrm{M}^{+}\right)}$ 563.2889, found 563.2868 .

\section{References}

1. (a) Grubbs, R. H., Ed.; Handbook of Metathesis, Vol. 1-3; Wiley-VCH: Weinheim, 2003. (b) Fürstner, A., Ed.; Topics in Organometallic Chemistry, Vol. 1; Springer-Verlag: Berlin, Heidelberg, 1998. For selected general reviews, see: (c) Vougioukalakis, G. C.; Grubbs, R. H. Chem. Rev. 2010, 110, 1746. (d) Nolan, S. P.; Clavier, H. Chem. Soc. Rev. 2010, 39, 3305. (e) Kotha, S.; Lahiri, K. Synlett 2007, 2767. (f) Chauvin, Y. Angew. Chem. Int. Ed. 2006, 45, 3741. (g) Schrock, R. R. Angew. Chem. Int. Ed. 2006, 45, 3748. (h) Grubbs, R. H. Angew. Chem. Int. Ed. 2006, 45, 3760. (i) Hoveyda, A. H.; Zhugralin, A. R. Nature 2007, 450, 243. (j) Nicolaou, K. C.; Bulger, P. G.; Sarlah, D. Angew. Chem., Int. Ed. 2005, 44, 4490. (k) Fürstner, A. Angew. Chem., Int. Ed. 2000, 39, 3012. (l) Grubbs, R. H.; Chang, S. Tetrahedron 1998, 54, 4413. (m) Schuster M.; Blechert S. Angew. Chem., Int. Ed. 1997, 36, 2037.

2. (a) Mori, M. Top. Organomet. Chem. 1998, 1, 133. (b) Kotha, S.; Meshram, M.; Tiwari, A. Chem. Soc. Rev. 2009, 38, 2065. (c) Hansen, E. C.; Lee, D. Acc. Chem. Res. 2006, 39, 509. (d) Diver, S. T.; Giessert, A. J. Chem. Rev. 2004, 104, 1317. (e) Poulsen, C. S.; Madsen, R. Synthesis 2003, 1. (f) Mori, M.; Sakakibara, N.; Kinoshita, A. J. Org. Chem. 1998, 63, 6082. (g) Kinoshita, A.; Mori, M. J. Org. Chem. 1996, 61, 8356.

3. Katz, T. J.; Sivavec, T. M. J. Am. Chem. Soc. 1985, 107, 737. 
4. (a) Kitamura, T.; Mori, M. Org. Lett. 2001, 3, 1161. (b) Mori, M.; Kuzuba, Y.; Kitamura, T.; Sato, Y. Org. Lett. 2002, 4, 3855. (c) Kitamura, T.; Kuzuba, Y.; Sato, Y.; Wakamatsu, H.; Fujita, R.; Mori, M. Tetrahedron, 2004, 60, 7375. (d) Mori, M.; Wakamatsu, H.; Tonogaki, K.; Fujita, R.; Kitamura, T.; Sato, Y. J. Org. Chem. 2005, 70, 1066. (e) Wakamatsu, H.; Sato, Y.; Fujita, R.; Mori, M. Heterocycles 2006, 67, 89. (f) Mori, M.; Wakamatsu, H.; Sato, Y.; Fujita, R. J. J. Mol. Catal. A: Chem. 2006, 254, 64. (g) Wakamatsu, H.; Sato, Y.; Fujita, R.; Mori, M. Adv. Synth. Catal. 2007, 349, 1231.

5. The combination of several metathesis transformations was refered as Ring Rearrangement Metathesis (RRM). For review of RRM, see: Holub, N.; Blechert, S. Chem. Asian J. 2007, 2, 1064.

6. White, B. H.; Snapper, M. L. J. Am. Chem. Soc. 2003, 125, 14901.

7. (a) Mitsunobu, O. Synthesis 1981, 1. (b) Hughes, D. L. Org. React. 1992, 42, 335.

8. (a) Sonogashira, K.; Tohda, Y.; Hagihara, N. Tetrahedron Lett. 1975, 16, 4467. (b) Chinchilla, R.; Nájera, C. Chem. Rev. 2007, 107, 874.

9. Sohn, J.-H.; Kim, K. H.; Lee, H.-Y.; No, Z. S.; Ihee, H. J. Am. Chem. Soc. 2008, 130, 16506. 